\title{
Complutum
}

ISSN: 1131-6993

\section{En busca del primer Homo: gestión de la investigación arqueológica en la Garganta de Olduvai (Tanzania)}

Manuel Domínguez-Rodrigo ${ }^{1}$, Marina Vegara-Riquelme ${ }^{2}$, Julia Aramendi ${ }^{3}$, Elia Organista ${ }^{4}$, Lucía CoboSánchez ${ }^{5}$, Lucía Hernández-Vivanco ${ }^{6}$, Fernando Diez-Martín ${ }^{7}$, José Manuel Maíllo-Fernández ${ }^{8}$, David Martín-Perea ${ }^{9}$, David Uribelarrea ${ }^{10}$ y Enrique Baquedano ${ }^{11}$

Resumen. Los yacimientos de la Garganta de Olduvai (Tanzania) constituyen un enclave único para el estudio de los primeros representantes del género Homo. La buena preservación de estos yacimientos y la cantidad de fósiles hallados en ellos posibilita que se sigan desarrollando excavaciones arqueológicas en este lugar. El equipo de investigación The Olduvai Paleoanthropology and Paleoecology Project (TOPPP), que trabaja en estos yacimientos desde 2006, promovió la construcción de la Estación Científica Aguirre-Mturi con el propósito de mejorar las condiciones laborales del personal investigador y de hacer del campamento un lugar seguro de trabajo. Durante el proceso de creación y construcción se tuvo en cuenta la salvaguarda de los materiales arqueológicos, y gracias a la existencia de un laboratorio en el campamento, es posible realizar tareas como la limpieza, el análisis y la restauración del material recuperado. En España, la creación del Instituto de Evolución en África (IDEA), sede actual del equipo TOPPP, nace con el objetivo de promover el estudio de la paleoantropología africana y el origen del ser humano en África. Junto a los trabajos de investigación, TOPPP realiza una intensa actividad divulgativa donde destaca la creación de exposiciones temporales y permanentes en España y Tanzania.

Palabras clave: Garganta de Olduvai, gestión, investigación, divulgación, Arqueología.

1 Instituto de Evolución en África (IDEA), Universidad de Alcalá, Covarrubias 36, 28010, Madrid, España. manuel.dominguezr@uah.es Universidad de Alcalá, Área de Prehistoria, Departamento de Historia y Filosofía, Colegios 2, 28801, Alcalá de Henares, España. Departamento de Antropología, Universidad de Rice, 6100 Main St., Houston, TX 77005-1827, USA.

2 Instituto de Evolución en África (IDEA), Universidad de Alcalá, Covarrubias 36, 28010, Madrid, España. marina.vegara@uah.es Universidad de Alcalá, Área de Prehistoria, Departamento de Historia y Filosofía, Colegios 2, 28801, Alcalá de Henares, España. Instituto de Evolución en África (IDEA), Universidad de Alcalá, Covarrubias 36, 28010, Madrid, España. juliaram@ucm.es Área de Prehistoria, Departamento de Prehistoria, Historia Antigua y Arqueología, Universidad Complutense, Profesor Aranguren s/n, 28040, Madrid, España.

4 Instituto de Evolución en África (IDEA), Universidad de Alcalá, Covarrubias 36, 28010, Madrid, España. elia.organista.labrado@ ofl.su.se

Laboratorio de Investigación Osteoarqueológica, Departamento de Arqueología y Estudios Clásicos, Universidad de Estocolmo, Wallenberglaboratoriet 10691 Estocolmo, Suecia.

5 Instituto de Evolución en África (IDEA), Universidad de Alcalá, Covarrubias 36, 28010, Madrid, España. cobosanchez.lucia@, gmail.com

6 Instituto de Evolución en África (IDEA), Universidad de Alcalá, Covarrubias 36, 28010, Madrid, España. lucia.hernandezv@uah.es Universidad de Alcalá, Área de Prehistoria, Departamento de Historia y Filosofía, Colegios 2, 28801, Alcalá de Henares, España.

7 Instituto de Evolución en África (IDEA), Universidad de Alcalá, Covarrubias 36, 28010, Madrid, España. fernando.diez.martin@uva.es Departamento de Prehistoria y Arqueología, Universidad de Valladolid, Plaza del Campus s/n, 47011, Valladolid, España.

8 Instituto de Evolución en África (IDEA), Universidad de Alcalá, Covarrubias 36, 28010, Madrid, España. jlmaillo@geo.uned.es Departamento de Prehistoria y Arqueología, Universidad Nacional de Educación a Distancia, UNED, Madrid, España.

9 Instituto de Evolución en África (IDEA), Universidad de Alcalá, Covarrubias 36, 28010, Madrid, España. davidmam@ucm.es Departamento de Paleobiología, Museo Nacional de Ciencias Naturales-CSIC, José Gutiérrez Abascal 2, 28006, Madrid, España. Área de Paleontología, Departamento de Geodinámica, Estratigrafía y Paleontología, Universidad Complutense, José Antonio Novais 12, 28040, Madrid, España.

10 Instituto de Evolución en África (IDEA), Universidad de Alcalá, Covarrubias 36, 28010, Madrid, España. uriben@ucm.es Área de Geodinámica Externa, Departamento de Geodinámica, Estratigrafía y Paleontología, Universidad Complutense, José Antonio Novais 12, 28040, Madrid, España.

11 Instituto de Evolución en África (IDEA), Universidad de Alcalá, Covarrubias 36, 28010, Madrid, España. enrique.baquedano@ madrid.org

Museo Arqueológico Regional de la Comunidad de Madrid, Plaza de las Bernardas s/n, 28801, Alcalá de Henares, Madrid. 
[en] In pursuit of the first Homo: Management of Archaeological Research in Olduvai Gorge (Tanzania)

\begin{abstract}
The sites at Olduvai Gorge (Tanzania) constitute a unique venue for the study of the first members of the genus Homo. The large amount of fossils recovered and the good state of preservation of these sites have boosted archaeological research in the Gorge throughout the years. The Olduvai Paleoanthropology and Paleoecology Project (TOPPP) research team, present at the Gorge since 2006, launched the construction of the Aguirre-Mturi Scientific Station within the limits of the Ngorongoro National Park with the aim of improving the working conditions and provide a safe working space. The safeguard of the archaeological materials was also one of the cornerstones during the design process and building of the station. The laboratory allows almost immediate cleaning, analysis and restoration of the materials recovered during field work. Meanwhile, in Spain, the Institute of Evolution in Africa (IDEA), current headquarters of the TOPPP team, was founded with the aim of promoting the study of African paleoanthropology and the origins of humankind in Africa. Along with the research work, TOPPP strives to bring the scientific knowledge to the general public in several ways, including the management of temporary and permanent exhibitions in Spain and Tanzania.
\end{abstract}

Keywords: Olduvai Gorge, archaeological management, research, scientific dissemination, Archaeology.

Sumario. Introducción. Las excavaciones en la Garganta de Olduvai (Tanzania). La construcción de la Estación Científica Aguirre-Mturi. El Instituto de Evolución en África (IDEA). El trabajo de divulgación científica del equipo. Perspectivas de futuro. Conclusiones. Agradecimientos. Bibliografía.

Como citar: Domínguez-Rodrigo, M. et al. (2021): En busca del primer Homo: gestión de la investigación arqueológica en la Garganta de Olduvai (Tanzania). Complutum, 32(2): 495-503.

\section{Introducción}

Los yacimientos de la Garganta de Olduvai, ubicados al norte de Tanzania, constituyen un enclave singular para el estudio de la Evolución Humana y del linaje evolutivo del género Homo. La Garganta de Olduvai, conocida también como la Cuna de la Humanidad, alberga en sus depósitos información de gran valor para comprender algunos aspectos fundamentales de la humanidad (Baquedano e Yravedra 2014). En este lugar fueron descubiertos los restos fósiles del holotipo de la especie Homo habilis (Leakey et al. 1964) y las primeras herramientas líticas que, por este motivo, fueron denominadas industria olduvayense (Leakey 1971). La preservación de estos yacimientos es excepcional, situándolos en un punto de referencia para el estudio de la paleoantropología africana. Los depósitos sedimentarios de la Garganta de Olduvai abarcan desde los 2.1 millones de años hasta el Holoceno (Uribelarrea 2017); y es durante el primer millón de años cuando tienen lugar los eventos más relevantes en relación con la aparición y evolución del género Homo en la garganta.

Desde el siglo pasado, han sido numerosos los profesionales que han trabajado en estos yacimientos y los hallazgos que se han producido en ellos. En la actualidad, un equipo internacional y multidisciplinar, The Olduvai Paleoanthropology and Paleoecology Project
(TOPPP), lidera los trabajos de investigación en la Garganta de Olduvai. El Instituto de Evolución en África (IDEA), codirigido por Manuel Domínguez-Rodrigo y Enrique Baquedano, es la sede central de este equipo de investigación.

Desde que en 2006 el equipo TOPPP comenzó sus trabajos en Olduvai, se han producido importantes avances científicos en el campo de la Evolución Humana que son imprescindibles para comprender mejor el pasado de la humanidad. Han sido descubiertos diversos yacimientos en el lecho más antiguo de la garganta como Philip Tobias Korongo (PTK), David Site (DS) o Alberto Gómez Site (AGS), lo que permite tener un conocimiento más preciso del comportamiento de los homininos que la habitaron entre 1.9 y 1.7 millones de años. Se han hallado nuevos fósiles de homininos, muy relevantes para completar el árbol filogenético humano, entre los que cabe destacar los siguientes: Olduvai Hominid $(\mathrm{OH})$ 80, el esqueleto parcial de un Paranthropus boisei hallado en el yacimiento Bell Korongo (BK) en el lecho II, gracias al cual se conoce mejor el esqueleto postcraneal de esta especie (Domínguez-Rodrigo et al. 2013); OH 86, una falange proximal con características modernas de un hominino diferente de Homo habilis y de Paranthropus boisei, hallada en el yacimiento PTK en el lecho I (Domínguez-Rodrigo et al. 2015), y $\mathrm{OH} 81$, dos fragmentos del parietal 
de un individuo infantil con hiperostosis porótica, en el yacimiento Sam Howard Korongo (SHK) del lecho II (Domínguez-Rodrigo et al. 2012). Los últimos hallazgos en el yacimiento Frida Leakey Korongo West (FLK West) han cambiado los paradigmas establecidos hasta el momento sobre el origen del tecnocomplejo Achelense (Diez-Martín et al. 2015). Y gracias al estudio tafonómico de los fósiles hallados en los nuevos yacimientos del lecho I, así como a la revisión de los ya estudiados a lo largo del siglo XX (Domínguez-Rodrigo et al. 2007), es posible conocer el agente o agentes formadores de los distintos yacimientos, la conducta de los primeros representantes del género Homo y de esta manera, dar respuesta a algunas de las grandes preguntas de la Evolución Humana.

\section{Las excavaciones en la Garganta de Olduvai (Tanzania)}

La preparación de las campañas de excavación que se realizan cada año en Olduvai es compleja, tanto por el coste económico que implica realizar estas tareas en un lugar tan alejado del acceso a recursos básicos como por la dificultad organizativa que todo ello conlleva. En este sentido, la existencia de una infraestructura próxima a los yacimientos que permita llevar a cabo actividades básicas en el transcurso de la excavación es fundamental. En la actualidad, el equipo TOPPP cuenta con la Estación Científica Aguirre-Mturi, pero no siempre ha sido así.

Al inicio de su andadura en Olduvai, los miembros del equipo se alojaban en tiendas de campaña junto a las casas de los guardas que custodian la Reserva del Ngorongoro y el Parque del Serengueti, y trabajaban en condiciones laborales e higiénicas precarias, sin seguridad sanitaria (Baquedano y DomínguezRodrigo 2014). El equipo no contaba con un lugar físico en el que poder organizar y revisar los materiales hallados durante la excavación, y todo ello limitaba las tareas imprescindibles que tenían que realizarse durante la campaña de trabajo.

Hoy el campamento del equipo TOPPP cuenta con una estación en la que se pueden acometer todo tipo de actividades básicas y necesarias durante el desarrollo de las campañas de excavación en los yacimientos de la Cuna de la Humanidad.

\section{La construcción de la Estación Científica Aguirre-Mturi}

En 2008 el equipo TOPPP propuso la solución al problema logístico al que hacía frente: se planteó crear un conjunto de estructuras estables que permitieran una mejora de las condiciones laborales y vitales del personal del equipo durante las campañas de excavación en la garganta. A través de un convenio con la ONG Cives Mundi y con el IDEA, la Estación Científica iba a estar gestionada por la Autoridad del Área de Conservación del Ngorongoro (NCAA) con la participación de la población local, que se beneficiaría también del proyecto (Baquedano y Domínguez-Rodrigo 2014). En esta empresa colaboró la Comunidad de Madrid en el ámbito económico y Cives Mundi en los aspectos organizativos. De esta manera, con el proyecto de la arquitecta Carla Mallol, en el año 2011 y tras los trámites pertinentes, comenzaron las obras de la Estación Científica Aguirre-Mturi.

La Estación Científica está compuesta por seis edificios independientes distribuidos por el campamento que se mimetizan en el paisaje sin tener un impacto visual fuerte (Figura 1). De entre este conjunto de estructuras, hay que destacar el laboratorio/almacén por su funcionalidad y su importancia para realizar actividades básicas de investigación (Figura 2). Se trata de un módulo cerrado dividido en dos partes que el equipo utiliza como almacén del material experimental y arqueológico que se recupera durante la excavación y como laboratorio. En este último, pequeño pero bien organizado, es donde se deposita el material arqueológico extraído en el campo para hacer una limpieza superficial y análisis preliminar del mismo, donde se ejecutan las actividades más urgentes de conservación-restauración, donde se procesan y vuelcan los datos recogidos durante el trabajo de campo a las bases de datos y, en definitiva, donde el equipo lleva a término todas aquellas tareas que se hacen imprescindibles durante la excavación de los yacimientos.

En este sentido, hay que mencionar la importancia de las tareas de conservación-restauración realizadas tanto en el campo como en el laboratorio. Estas dependerán del estado de conservación en el que se encuentren los materiales, así como de las necesidades más urgentes del equipo de investigación durante la campaña en cuestión (Hernández-Vivanco 2020).

Junto al almacén/laboratorio destaca un edificio multifuncional, abierto por sus lados 


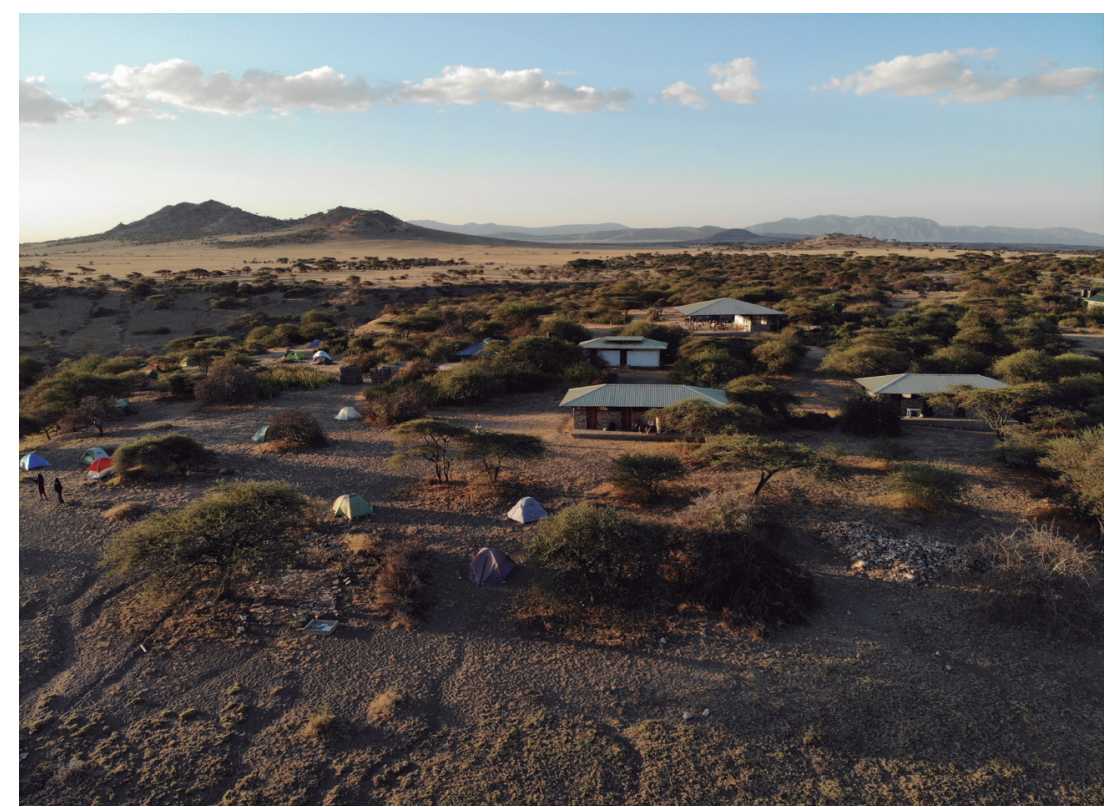

Figura 1. Estación Científica Aguirre-Mturi y campamento del equipo TOPPP. Foto: Javier Trueba.

y solo cubierto en la zona superior, donde se ubican tanto la cocina como el comedor, que también se usa como sala de conferencias y reuniones. El resto de la Estación Científica está conformada por dos edificios para alojamiento compuesto cada uno por cuatro habitaciones dobles y otros dos edificios de servicios con duchas, inodoros, etc.

La superficie total construida es de 509,9 $\mathrm{m}^{2}$ y se utiliza un sistema de energía solar que abastece todas las instalaciones de la Estación Científica. Este conjunto de estructuras, construidas con materiales locales que se adaptan al entorno en el que se encuentran, dotan al campamento de unas prestaciones básicas y al equipo de las condiciones necesarias para poder desarrollar el trabajo de investigación. De la misma manera, el barracón principal es el enclave de reunión e interacción en el que el equipo pasa la mayor parte del tiempo cuando no está trabajando. Desde este lugar, abierto al aire libre, se puede ver al norte Naibor Soit, lugar en el que los homininos se abastecían de materia prima para sus herramientas $\mathrm{y}$, al oeste, una reconfortante puesta de sol tras un día de trabajo en los barrancos de la garganta.

El nombre dado a la infraestructura simboliza la hermandad hispano-tanzana existente desde 2006 hasta la actualidad para el desarrollo de los trabajos de investigación en la Garganta de Olduvai. Con el nombre de la Estación Científica, llamada Aguirre-Mturi, se pretendió homenajear a estos dos grandes referentes del mundo de la Paleontología y de la Arqueología respectivamente. Emiliano Aguirre es el padre de la paleoantropología española y fue el primer paleontólogo español que visitó la Garganta de Olduvai en 1968, donde estuvo en contacto con la familia Leakey; por su parte, Amin Mturi, profesor de Arqueología, fue uno de los pioneros de esta rama de conocimiento en Tanzania y compañero de los Leakey (Baquedano y Domínguez-Rodrigo 2014).

\section{El Instituto de Evolución en África (IDEA)}

La creación del IDEA tiene lugar en 2010 y el objetivo principal de este centro es el estudio de la paleoantropología africana y el origen del ser humano en África. El IDEA forma parte de un consorcio constituido por el Museo Arqueológico Regional de la Comunidad de Madrid y la Universidad de Alcalá de Henares, y depende académicamente de esta última y de la Fundación General de la Universidad de Alcalá. En la actualidad, este instituto tiene su sede en la calle Covarrubias 36 (Madrid), y en sus fondos, alberga una colección experimental neotafonómica de macromamíferos de referencia a nivel internacional y una biblioteca especializada en Arqueología africana.

En el IDEA se realizan diversas tareas de investigación y como sede del equipo TOPPP, 


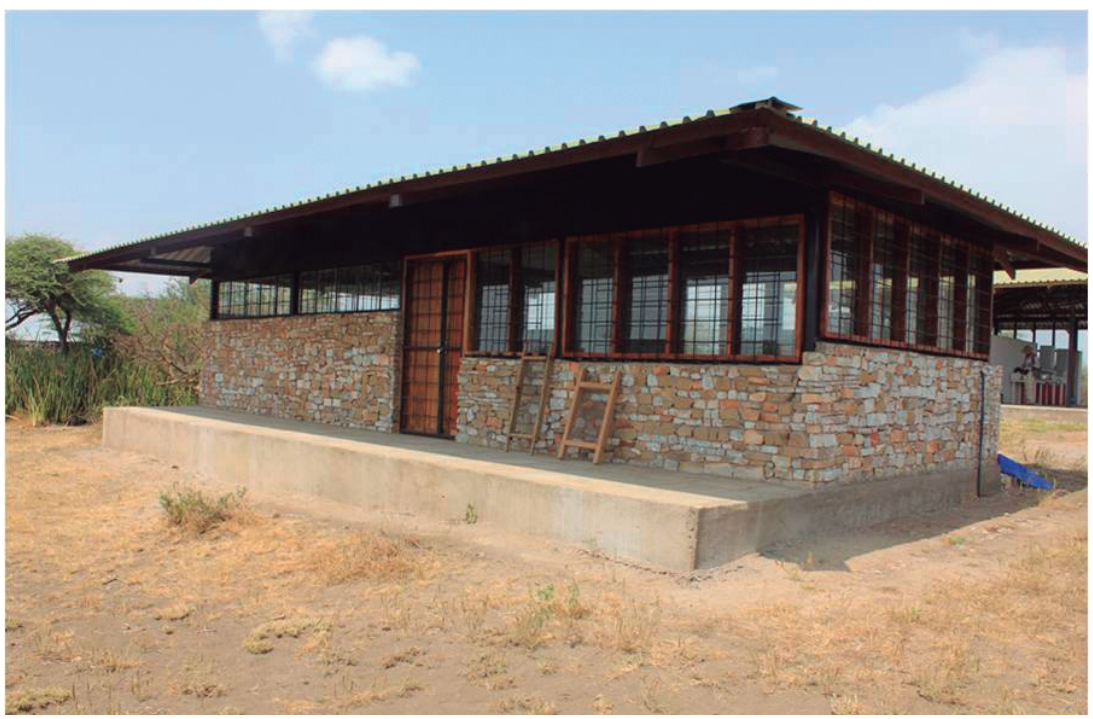

Figura 2. Laboratorio y almacén de la Estación Científica Aguirre-Mturi. Foto: Javier Trueba.

ofrece un lugar de trabajo para todos los miembros de este. Las instalaciones del IDEA también han sido disfrutadas por investigadores de otras instituciones de prestigio que han realizado estancias de investigación en este centro, como Daniel Lieberman de la Universidad de Harvard, Charles Egeland de la Universidad de Carolina del Norte o Erik Otarola Castillo de la Universidad de Purdue. En la actualidad, es un centro de investigación que está generando una gran cantidad de publicaciones científicas de impacto (112 artículos hasta el momento).

Son 10 las Tesis Doctorales defendidas por miembros del equipo TOPPP y numerosas las que están en curso, así como los Trabajos Fin de Grado y Fin de Máster defendidos por miembros del equipo de investigación. No obstante, junto a las labores de investigación, el IDEA también nace con el objetivo de ser un centro de formación especializado en disciplinas como la Tafonomía, la Arqueología, la Paleoecología, la Antropología, la Estadística Multivariante o la Inteligencia Artificial. En relación con este último campo, el IDEA es un centro pionero en la aplicación de herramientas de Inteligencia Artificial en los estudios de Evolución Humana, lo que lo convierte en un centro de referencia a nivel internacional.

Junto a los trabajos de investigación y formación que se realizan en las instalaciones del IDEA, este instituto y la Universidad de Carolina del Norte coordinan una escuela de campo en los yacimientos de la Garganta de Olduvai, lo que permite que alumnos internacionales se formen en disciplinas como la
Arqueología, la Geología, la Tafonomía o la Paleoantropología.

\section{El trabajo de divulgación científica del equipo}

El equipo de investigación TOPPP considera imprescindible transmitir a la sociedad el conocimiento obtenido a raíz de los trabajos de investigación que se realizan en los yacimientos de la Garganta de Olduvai. De esta manera, junto a las publicaciones en revistas científicas de impacto, el equipo realiza actividades divulgativas a nivel internacional con el objetivo de difundir entre la sociedad el conocimiento que producen sus investigaciones en la garganta. Esta actividad se realiza mediante diversas vías entre las que destacan: el uso de las redes sociales, la participación en medios de comunicación (televisión, radio y prensa), los documentales y las exposiciones tanto temporales como permanentes. En este sentido, hay que destacar la extensa y rigurosa exposición temporal titulada La Cuna de la Humanidad, cuyos comisarios fueron Manuel DomínguezRodrigo y Enrique Baquedano.

Esta exposición, organizada por el IDEA, el Museo Arqueológico Regional de la Comunidad de Madrid, el Museo de la Evolución Humana de la Junta de Castilla y León y el Museo Nacional y Casa de Cultura de Dar es Salaam, fue inaugurada en 2014 en el Museo Arqueológico Regional en Alcalá de Henares, desde donde viajó al Museo de la Evolución Humana en Burgos y al Museo de Ciencias Cosmo- 
Caixa en Barcelona (Figura 3). Finalmente, los materiales y las réplicas de la exposición fueron llevadas al Museo Nacional de Dar es Salaam donde forman parte en la actualidad de su exposición permanente. Esta exposición, inaugurada en enero de 2018, fue readaptada al espacio disponible en este museo; está compuesta por fósiles originales, réplicas, material audiovisual de Javier Trueba, ilustraciones de Mauricio Antón y cartelas explicativas de las diferentes vitrinas que, en conjunto, permiten acercar al visitante a un pasado remoto.

El objetivo principal de la exposición temporal La Cuna de la Humanidad era acercar el pasado de la humanidad al espectador y trasladarlo al continente africano, lugar donde surgieron los primeros representantes del linaje evolutivo del género Homo.

Previa a esta exposición tuvo lugar una campaña de restauración en 2012 con el objetivo de preparar adecuadamente los fósiles para su exhibición. Para ello, primero se realizó una visita a los fondos del Museo de Dar es Salaam con el objetivo de examinar el estado de conservación de los fósiles que se pretendía exportar. Una vez seleccionados los materiales y trasladados al Museo Arqueológico Regional, se procedió a realizar las labores de limpieza y conservación-restauración previas a la exhibición de las piezas.

Entre las piezas que formaron parte de esta exposición destacan una gran colección de fósiles originales de fauna africana extinta, fó- siles de homininos originales como $\mathrm{OH} 80$, varias reproducciones de fósiles humanos y diversos utensilios originales de industria lítica. El contenido de la exposición se recogió en la edición de un catálogo de dos volúmenes con el título de la propia exposición y con textos en español e inglés, que recogen diversos capítulos redactados por investigadores de prestigio en el campo de la Evolución Humana.

En 2017, el equipo TOPPP también se ocupó del contenido de la exposición para el nuevo Museo de la Garganta de Olduvai, cuya construcción fue posible gracias a la ayuda de la Unión Europea. La exposición que se preparó para este museo está basada en la de $L a$ Cuna de la Humanidad, y contiene fósiles originales y duplicados de las secciones más relevantes de la misma. Esta exposición se inauguró a finales del año 2017 en una ceremonia de apertura a la que asistió la vicepresidenta de Tanzania, Samia Suluhu Hassan, aunque finalmente fue abierta al público en 2018.

La exposición es la culminación del compromiso de TOPPP por divulgar entre la comunidad local y la foránea que se acerca a Olduvai, que se había iniciado anteriormente en el 2009, con la elaboración de un panel ilustrativo realizado por Manuel López Herrero de la reconstrucción del modo de vida de los primeros seres humanos, colocado en el mismo yacimiento emblemático de FLK Zinj, como motivo del 50 aniversario del descubrimiento de la especie de Paranthropus boisei, que hizo

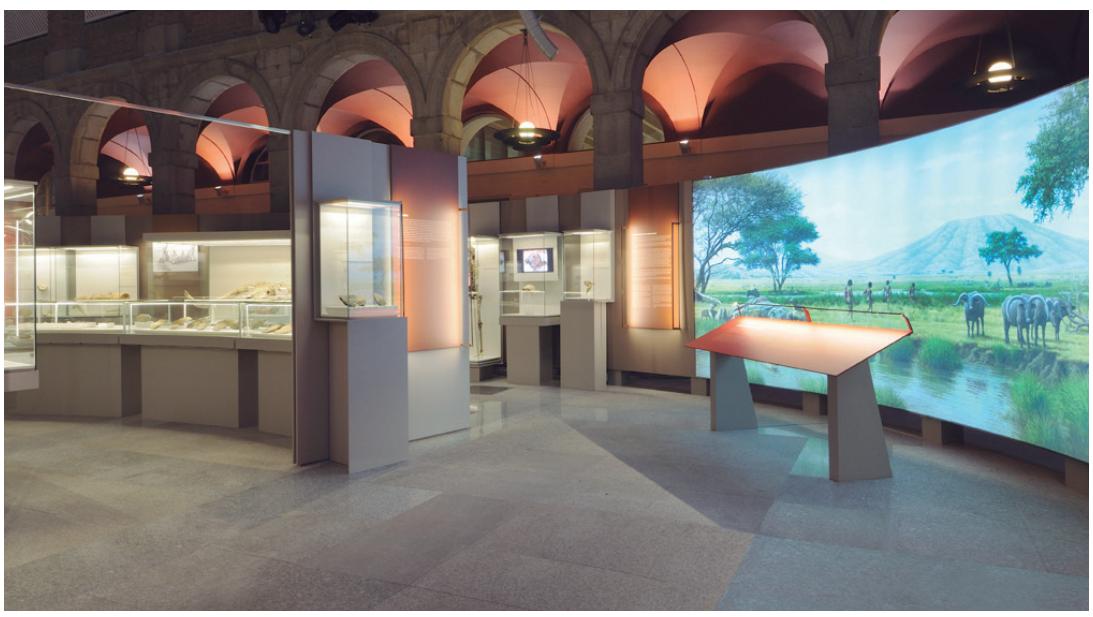

Figura 3. Vista de parte de la exposición temporal La Cuna de la Humanidad en el Museo Arqueológico Regional de la Comunidad de Madrid: a la derecha, uno de los paneles de gran formato con una ilustración de Mauricio Antón y en el centro y a la izquierda, diversas vitrinas con las piezas expuestas. Foto: Mario Torquemada. 
famosa a la garganta en todo el mundo. Dicha celebración tuvo lugar en forma de congreso internacional en la ciudad de Arusha y posterior desplazamiento de las autoridades a Olduvai para inaugurar oficialmente dicho panel emblemático (Figura 4). Gracias a este panel, se reactivaron las visitas guiadas al interior de la garganta, no siendo ahora el museo el destino final y único de los visitantes.

El Museo de Olduvai se encuentra dentro del Área de Conservación del Ngorongoro, al borde de la Garganta de Olduvai; desde el mirador del museo, se puede apreciar una panorámica de la garganta con el famoso Castillo de Olduvai en el centro y el campamento del equipo TOPPP y Naibor Soit al fondo. El museo original fue levantado en la década de los años 70 por Mary Leakey y más tarde pasó a ser gestionado por las autoridades tanzanas. En los años 90 fue ampliado por el Paul Getty Conservation Institute y en el año 2017 se inició la construcción del nuevo museo junto a la estructura inicial (Figura 5). La forma circular del museo está inspirada en una boma masái y el edificio está construido con materiales que se mimetizan en el paisaje de la zona.

Junto a la exposición de La Cuna de la Humanidad, el Museo Arqueológico Regional inauguró en 2018 otra exposición temporal sobre uno de los yacimientos del lecho II de la Gar- ganta de Olduvai: FLK West, donde se halló en el año 2015 un bifaz único tallado en basalto cuyo descubrimiento aportó información de gran relevancia sobre el origen del tecnocomplejo Achelense. El comisario de esta exposición titulada En África hace 1,7 millones de años: el origen del Achelense fue Fernando Diez-Martín.

En la actualidad TOPPP está colaborando con el ministerio de Recursos Naturales y Turismo tanzano y especialmente con las autoridades del Área de Protección del Ngorongoro para la inclusión de Olduvai en el área mayor del Geoparque del norte de Tanzania, auspiciado por la UNESCO.

\section{Perspectivas de futuro}

El objetivo del equipo TOPPP es seguir realizando campañas de excavación en los yacimientos de la prestigiosa Garganta de Olduvai, que destacan por su magnífica preservación como consecuencia del entorno en el que se encuentran. Continuar con los trabajos de investigación en estos yacimientos es fundamental para aportar nuevos datos a las grandes cuestiones de la Evolución Humana. Por un lado, terminar de excavar los nuevos yacimientos del lecho I y realizar el análisis del

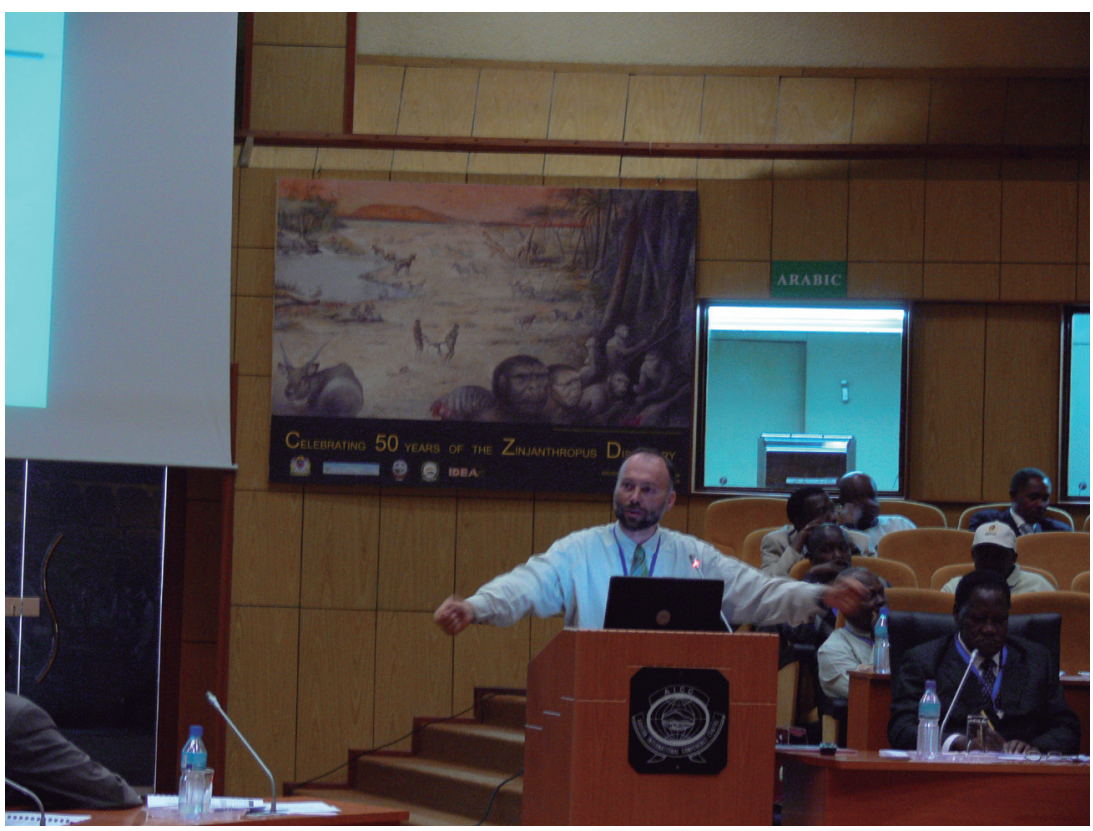

Figura 4. Manuel Domínguez-Rodrigo en el congreso internacional celebrado en Arusha en 2009; al fondo, el panel colocado en el yacimiento FLK Zinj.

Foto: Enrique Baquedano. 


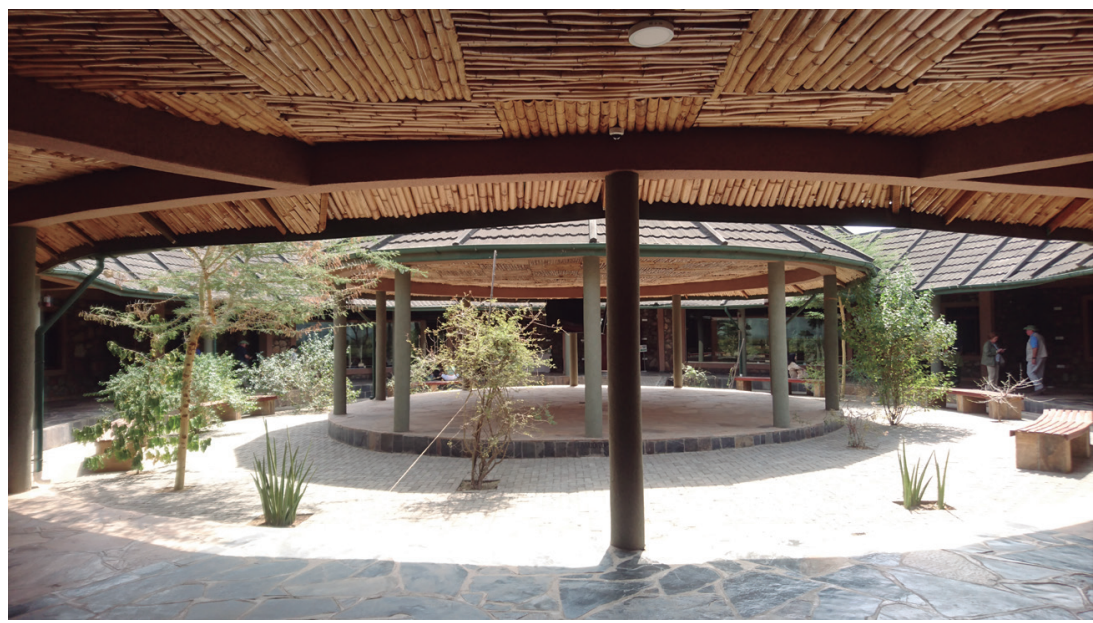

Figura 5. Vista del interior del Museo de la Garganta de Olduvai. Foto: David Uribelarrea.

material aplicando herramientas informáticas como la Inteligencia Artificial, permitirá ampliar el conocimiento que sobre los primeros representantes del género Homo se tiene en la actualidad. Por otro lado, el estudio de los yacimientos achelenses del lecho II de la Garganta de Olduvai es fundamental para conocer mejor a los grupos humanos que desarrollaron esta tecnología y lo que ello conlleva.

Junto al estudio de la fauna y de la industria lítica recuperada en estos yacimientos, serán relevantes los resultados que se obtengan tras el análisis de los homininos desde un punto de vista biomecánico y tafonómico.

Al mismo tiempo, el equipo pretende seguir poniendo en valor el patrimonio tanzano y la población local del entorno de la Garganta de Olduvai, y seguir realizando una profusa labor de divulgación científica, con el fin de acercar a la sociedad el conocimiento sobre el pasado de la humanidad ${ }^{12}$.

\section{Conclusiones}

Desde que en 2006 un equipo hispano-tanzano comenzó sus trabajos en la Garganta de Olduvai, han sido numerosos los avances que se han producido en el campo de la Evolución Humana; la construcción de la Estación Científica Aguirre-Mturi ha sido fundamental para mejorar las condiciones de los investigadores en Ol-

12 En este sentido, cabe mencionar la gran labor que la Dra. Nines Querol ha realizado a lo largo de su trayectoria profesional, destacando sus trabajos en el campo de la teoría sobre Gestión del Patrimonio, los Parques Arqueológicos y el género en la Arqueología. duvai, y en España, la creación y consolidación del IDEA, asegura la continuidad de la investigación en el campo de la Evolución Humana en África, al tiempo que contribuye a la formación de especialistas en esta área de conocimiento. Para el equipo TOPPP, es un deber social y moral transmitir y devolver a la sociedad el conocimiento que produce como resultado de las investigaciones que se realizan en la Garganta de Olduvai, que alberga los yacimientos arqueológicos más ricos sobre el origen del ser humano.

\section{Agradecimientos}

A la Comisión para la Ciencia y la Tecnología (COSTECH), a la Autoridad del Área de Conservación del Ngorongoro (NCAA) y al Departamento de Antigüedades y al Ministerio de Turismo y Recursos Naturales en Tanzania por el permiso para investigar en la Garganta de Olduvai. Al Ministerio de Ciencia, Innovación y Universidades por el proyecto concedido (HAR2017-82463-C4-1-P) y por la financiación para los contratos predoctorales de Formación de Profesorado Universitario (FPU) de MVR y JA; al Ministerio de Cultura a través del Instituto de Patrimonio Cultural de España por las ayudadas concedidas para Proyectos Arqueológicos en el Exterior, y a la Fundación Palarq por las ayudas para apoyar las Misiones de Arqueología y Paleontología Españolas en el en el extranjero. También agradecemos especialmente a todos los compañeros de campo tanzanos su trabajo.

Esta contribución forma parte del homenaje a M. ${ }^{\mathrm{a}}$ Ángeles Querol. MDR desea rendir tributo de esta manera a la que fue su profesora 
y directora de tesis, y bajo cuyo tutelaje puso el cimiento de su trayectoria académica. Los años en los que trabajó bajo su amparo fueron de mucho aprendizaje y disfrute. Ella fue el único amparo académico que tuvo en esos años difíciles y su apoyo fue fundamental en la lucha contra los elementos que fue el periodo de conexión y anclaje con la universidad Complutense como profesor. "Nines, por todo lo que hiciste por mí, me complace hoy poder rendirte merecido homenaje por tu trayectoria profesional y por el legado que dejas".

\section{Bibliografía}

Baquedano, E.; Domínguez-Rodrigo, M. (2014): La estación "Emiliano Aguirre”. En: M. DomínguezRodrigo y E. Baquedano, eds., La Cuna de la Humanidad. Volumen I. BOCM: 89-95.

Baquedano, E.; Yravedra, J. (2014): Olduvai Gorge. La Cuna de la Humanidad. En: M. Domínguez-Rodrigo y E. Baquedano, eds., La Cuna de la Humanidad. Volumen II. BOCM: 153-171.

Diez-Martín, F.; Sánchez P.; Uribelarrea, D.; Baquedano, E.; Mark, D.; Mabulla, A.; Fraile, C.; Duque, J.; Díaz, I.; Pérez-González, A.; Yravedra, J.; Egeland, C.; Organista, E.; Domínguez-Rodrigo, M. (2016): The origin of the Acheulean: The 1.7 Million-year-old site of FLK West, Olduvai Gorge. Nature Scientific Reports, 5, 17839. https://doi.org/10.1038/srep17839

Domínguez-Rodrigo, M.; Barba, R.; Egeland, C. (2007): Deconstructing Olduvai: A Taphonomic Study of the Bed I Sites. Springer. https://doi.org/10.1007/978-1-4020-6152-3

Domínguez-Rodrigo, M.; Pickering, T. R.; Almécija, S.; Heaton, J. L.; Baquedano, E.; Mabulla, A.; Uribelarrea, D. (2015): Earliest modern human-like hand bone from a new 1.84-million-year-old site at Olduvai in Tanzania. Nature Communication, 6, 7987. https://doi.org/10.1038/ncomms8987

Domínguez-Rodrigo, M.; Pickering, T. R.; Baquedano, E.; Mabulla, A.; Mark, D. F.; Musiba, C.; Bunn, H. T.; Uribelarrea, D.; Smith, V.; Diez-Martin, F.; Pérez-González, A.; Sánchez, P.; Santonja, M.; Barboni, D.; Gidna, A.; Ashley, G.; Yravedra, J.; Heaton, J. L.; Arriaza, M. C. (2013): First Partial Skeleton of a 1.34-million-year-old Paranthropus boisei from bed II, Olduvai Gorge, Tanzania. PLoS ONE, 8 (12), e80347. http://dx.doi.org/10.1371/journal.pone.0080347

Domínguez-Rodrigo M.; Pickering T. R.; Diez-Martín F.; Mabulla A.; Musiba C.; Trancho, G.; Baquedano, E.; Bunn, H. T.; Barboni, D.; Santonja, M.; Uribelarrea, D.; Ashley, G. M.; Martínez-Ávila, M. S.; Barba, R.; Gidna, A.; Yravedra, J.; Arriaza, C. (2012): Earliest Porotic Hyperostosis on a 1.5-MillionYear-Old Hominin, Olduvai Gorge, Tanzania, PLoS ONE. 7 (10), e46414. https://doi.org/10.1371/ journal.pone.0046414

Hernández-Vivanco, L. (2020): Limpieza y preparación de los fósiles para su estudio y exhibición: la restauración de los fósiles en los yacimientos de la Garganta de Olduvai (Tanzania) y Pinilla del Valle (Madrid, España). En: S. Domínguez-Solera, coord., Cuando Empezábamos a ser nosotr@s: curso sobre el Paleolítico Inferior y Medio a nivel mundial (Vol. II), Diputación de Cuenca, Cuenca: 53-66.

Leakey, L. S. B.; Tobias, P. V.; Napier, J. R. (1964): A new species of the genus Homo from Olduvai Gorge. Nature, 202: 7-9.

Leakey, M. D. (1971): Olduvai Gorge, 3. Excavations in Beds I and II, 1960-1963. Cambridge University Press, Cambridge.

Uribelarrea, D. (2017): Geología en la Cuna de la Humanidad. Cómo trabaja un geólogo en los yacimientos de la garganta de Olduvai (Tanzania). Enseñanza de las ciencias de la tierra, 25 (1): 74-81. 
